\title{
A Competitive Duopoly where Information Spillovers can be Mutually Advantageous
}

\author{
Thierry Lafay ${ }^{* *}$
}

\section{Introduction}

On many markets, firms are not identical. They sell different products, their sizes are different and they have different market shares. If models such as [6] may explain the heterogeneity between products, the heterogeneity of firm' sizes and market shares remains quite unexplained when the products are homogenous. Indeed, if we think about a quantity competition, [4] predicts a symmetric equilibrium. Of course, [15] emphasized the fact that different commitment abilities change the nature of equilibrium, but the origins of these differences remain unclear. If models such as [1] and [16] explain market shares asymmetries by cost asymmetries, the question remains: why would firms have ex ante different costs ?

Our study concerns the case of symmetric ex ante firms. In such a setting with a discrete two-period model, [9] give conditions for an endogenous Stackelberg Equilibrium to arise ${ }^{1}$. [11] gives an example of an endogenous leadership in an investment game with an uncertainty on the profitability of the market. However, it is shown in [14] and in [3] that a sequential move is not likely to be an equilibrium in a simple two-period linear model with demand uncertainty. The reason is that, with quantity competition, the industrial surplus is usually higher when firms produce simultaneously rather than sequentially. Thus, the preempted firm has a strong incentive to deviate from the sequential move. [10] show in a discrete time model under uncertainty that the existence of asymmetric equilibria depends on the information revealed to the followers. In a continuous time model, [7] give also conditions such that a natural Stackelberg solution emerges. Moreover, the equilibrium is such that there is a rent equalization in a duopoly setting.

The author thanks two anonymous referees and Jean Pierre Ponssard for the time he spent in the elaboration of the model, for his comments and useful advices.

* PRISM-Sorbonne, Université Paris 1 Panthéon-Sorbonne, 17 rue de la Sorbonne, 75231 Paris. Email: tlafay@univ-paris1.fr

1 In the "qualitative game" the sequential output has to Pareto dominate the simultaneous output. 
Since demand creates its own supply, we want to understand in this paper, when and how supply emerges when firms are ex ante identical. For example, in the cement industry, when an emerging market is expected to grow each year, firms need at some time to build a new factory in order to serve the extra demand. Firms would prefer to build this factory as late as possible however, a competitor might build a factory earlier and preempt its natural market share. Moreover, when a competitor builds a factory there are information spillovers such that the follower can delay its investment, thereby gathering more information on the market demand.

In our model, we assume that future demand happens at time zero. Firms can produce quantities in order to serve this demand at any time $t$ before time zero. There is a gain for preemption (the leader becomes a Stackelberg leader), and a cost due to an early choice of production (costs are engaged when producing and there is a discount rate). In this continuous-time framework à la [7], we explicit the profit functions by using a linear model of quantity competition. Our assumptions regarding the information on demand are close to [10] but we do not introduce an explicit multi-period market. Moreover, we assume that there might be information spillovers between the leader and its follower which create second mover advantages ${ }^{2}$. For example, leaders usually face uncertainty about the demand function (the level of demand may not be well estimated, the first products may not be well designed for these new buyers) and also about the production costs (on new markets, the information on labor cost or any other input costs may be unknown to leaders but disclosed to followers). Followers can also usually learn from leaders' past actions. Indeed, if firms own uncorrelated information about the demand function, then the leader will at least reveal some pieces of its private information by producing.

This paper presents two main results. First, we show that, when the market demand is publicly known there is a classical preemption race: firms produce too soon and supply the future demand earlier than necessary. Second, with market uncertainty, different equilibria may arise, depending on the information spillovers between a leader and a follower. Without information spillovers, most of the literature conclude that the equilibrium is symmetric and that the preemption race implies a rent dissipation. On the contrary, our results emphasize the fact that, with information spillovers, the equilibrium can be asymmetric and the leader's rent is not fully dissipated. Whatever the equilibrium, firms ex ante earn the same expected profits: the industry surplus is equally shared between firms so that there is a 'rent equalization' as described in [7]. As a conclusion, the adaptation of the usual rent dissipation result in an uncertain context should be cautiously made. Indeed, the sequential equilibrium in our model may imply

2 In fact, these first versus second mover advantages can also explain the industry cluster natural formation(see [13]). 
higher payoff than the Cournot one with no pre-commitment. Hence, if information spillovers obviously serve the follower's interests, it can also even serve the leader's interests. A natural sequential entry on the market can emerge and be Pareto-superior compared to a case where firms engage in a preemption race.

Section 2 introduces the basic model that exhibits a preemption race. In Section 3, we introduce demand uncertainty and derive the equilibria of the game when there are information spillovers between a leader and a follower. Section 4 discusses the results and Section 5 eventually concludes.

\section{The Model with perfect information}

The model is a canonical duopolistic model. There are two firms and they compete on quantity. We use an inverse demand function $P=a-b$. $Q$ where $P$ is the market price and $Q$ is the total quantity produced. The firms are initially symmetric. The production cost on the market is $c$. Hence, this is a classical linear model of quantity competition known as the Cournot Nash game. We then allow firms to pre-commit before time 0 which is the time when market clears. For convenience, time goes backward from $t=\infty$ to $t=0$. Hence larger times indexes are indeed sooner times. The key decision is when to produce.

There is a discount factor such that the first best is to produce as late as possible. For simplicity's sake, profits are actualized at time 0 , when the market clears, using an ex ante discount factor $0<\frac{1}{\delta}<1^{3}$. In order to make the commitments of firms credible, we assume that firms can only produce once $^{4}$. They can neither decrease nor increase their production through time. Moreover they can always get a zero profit by not entering the market.

Alternatively, the model could also be seen as a competition on capacity where the unit cost of setting up new capacity is $c$. In this case, either we may think that the marginal cost of producing in a plant is 0 (but we still may have to give an additional condition in order to prove that firms produce up to their capacity) or we may see the price as the average revenue of capacity. Indeed, the inverse demand function is the simplest function that exhibits two basic properties: the average revenue decreases with decision variables, and decision variables are strategic substitutes. Thus, any type of investment game that presents those properties can fit our model.

3 Note that $\delta>1$. For example, producing at time zero a quantity $q$ costs $c q$, whereas at a time $t$ before time 0 , it costs $c \delta^{t} q$ when you actualize at time zero.

4 See [12] for a model introducing both multiple investment and time-to-build problems. 
Figure 1. Game Sequence

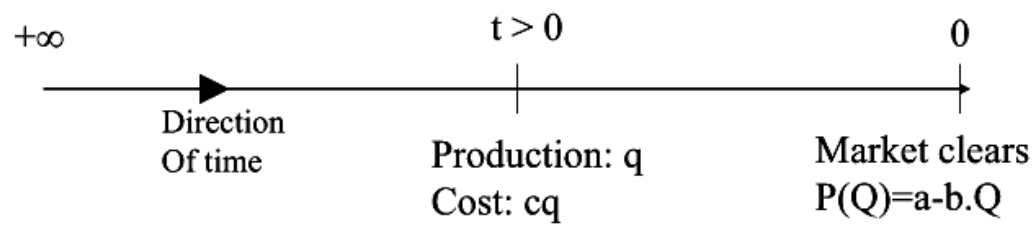

The timing of the game is represented in Fig. 1. This is a stopping game where each firm has to choose a stopping date at which it decides a quantity to produce.

Two situations may arise: -either one firm produces before the other and then becomes a Stackelberg leader, -or both firms produce simultaneously at time $t$ and then produce the Cournot quantities.

We assume that, when one firm decides to produce at time $t$, then it instantaneously learns if the other firm also decided to produce, prior to choose its quantity. Hence, there is no Stackelberg warfare if both firms produce simultaneously.

A pseudo discrete version of the stopping game is represented in Fig. 2.

Figure 2. The 'discrete' version of the game

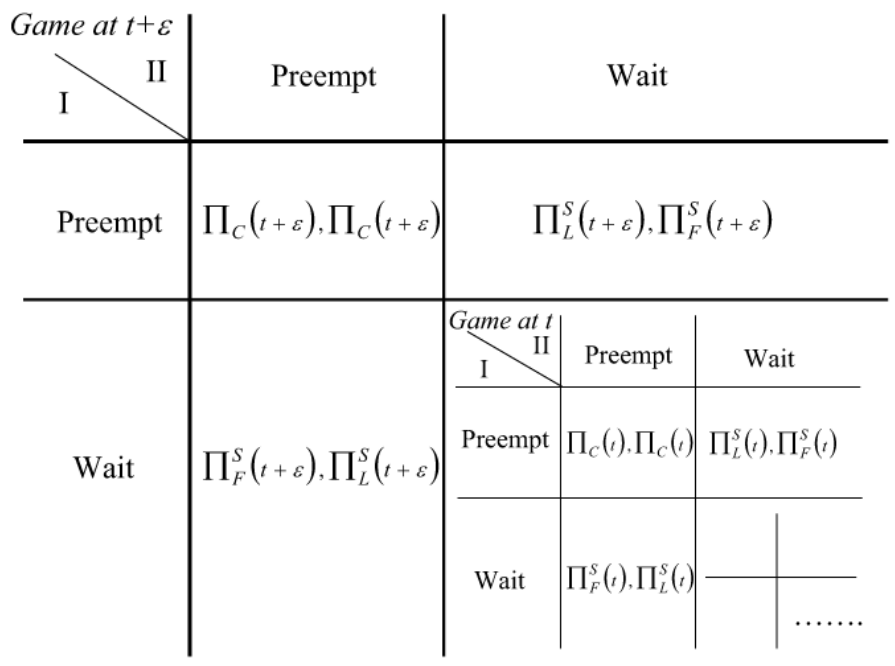

As a notation $q_{L}^{S}$ and $q_{F}^{S}$ represent respectively the leader's quantity and the follower's one in case of a Stackelberg game, $q^{C}$ represents the Cournot quantity. We use the same notation for the profits i.e. $\Pi_{L}^{S}, \Pi_{F}^{S}$ and $\Pi^{C}$. 
We also denote $\Pi_{0}^{C}$ as the firms' profits when they produce the classical Cournot quantities at time 0 , that is when market clears. Moreover, we assume that $a>c$ (otherwise the market is never profitable). We focus on the subgame perfect equilibrium in pure strategies of our games.

\subsection{Subgames}

Let $t$ be the production time when the first firm produces on the market.

We have a Stackelberg game at time $t$ when one firm decides to produce before its competitor. It is obvious that the second firm is going to produce only at the end of the game. The leader produces then $q_{L}^{S}=\frac{a+c-2 . c . \delta^{t}}{2 . b}$ while the follower produces $q_{F}^{S}=\frac{a-3 \cdot c+2 \cdot c \cdot \delta^{t}}{4 \cdot b}$. Both firms get the following profits: $\Pi_{L}^{S}(t)=\frac{1}{8 . b}\left(a+c-2 \cdot c \cdot \delta^{t^{2}}\right)^{2}$ and $\Pi_{F}^{S}(t)=\frac{1}{16 . b} \cdot\left(a-3 \cdot c+2 \cdot c \cdot \delta^{t}\right)^{2}$. There is a threshold time $\bar{t}=\frac{1}{\ln (\delta)} \cdot \ln \left(\frac{a+c}{2 . c}\right)$ such that, if $t>\bar{t}$, then a Stackelberg leader cannot enter the market as it would produce too early and would not cover its costs. The time $\bar{t}$ is defined as the largest time such that the leader makes a non-negative profit on the market.

In a Cournot subgame, firms enter the market at the same time $t$. They maximize their profits: $\max _{q_{i}}\left[\left(a-b . q_{i}-b . q_{j}\right) q_{i}-c . \delta^{t} . q_{i}\right]$. The Nash equilibrium is such that firms produce the same quantities $q^{C}=\frac{a-c . \delta^{t}}{3 . b}$ and get $\Pi^{C}(t)=\frac{\left(a-c . \delta^{t}\right)^{2}}{9 . b}$.

\subsection{Classical preemption race}

We now look for the subgame perfect equilibrium in pure strategies of the extensive game, where firms choose first the production timing and then choose the output knowing whether the other firm chose the same time of production. This game corresponds to the observable delay version of a preemption game ${ }^{5}$ in a continuous time model. Firms can observe past production and, at each time, firms decide whether or not to produce. When one firm decides to produce, this piece of information becomes public to the other, and firms decide which quantity to produce. We chose this kind of game in order to have comparable results with the classical literature on the trade-off between commitment and flexibility ${ }^{6}$. The alternative way of modeling, the "action commitment" game ${ }^{7}$, would typically have no equilibrium in pure strategies.

5 See [9] for the exact definition of those kind of game.

6 See for example [14].

7 In this version of the game, firms would no longer announce their stopping strategy prior to choose its quantity. 
Proposition 1. The only subgame perfect equilibrium in pure strategies of the model with perfect information is such that both firms produce at the same time $t^{*}$, defined by $\Pi_{L}^{S}\left(t^{*}\right)=\Pi^{C}\left(t^{*}\right)$.

This result implies that there is a leader's rent dissipation in the sense that the extra gain of a leader vanishes for both firms ${ }^{8}$. If we compare this equilibrium to the case where no firm pre-commits, we may remark that this pre-commitment of both firms is made for defensive reasons rather than aggressive ones. Indeed, in this pre-commitment game, both firms earn less by pre-committing than they would if they had not the opportunity to do so. We conclude that the preemption rent is fully dissipated, furthermore both firms are worse off.

Figure 3. The preemption race

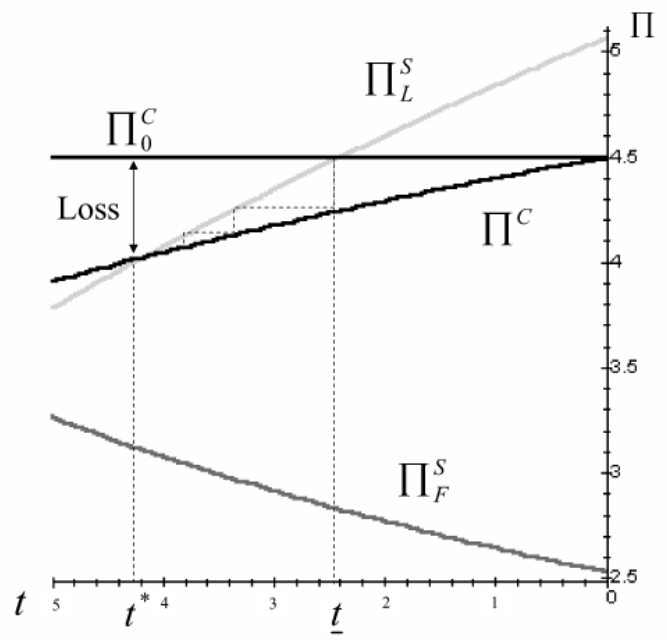

Figure 3 shows that the preemption race not only dissipates the leader's rents but also that firms lose more than the preemption profit ${ }^{9}$ by producing earlier than the market (this is of course due to the immobilization of capital). The preemption race works as it is drawn on Fig. 3. As soon as a leader earns more than what it will earn if it produces at time 0 , both firms have an incentive in pre-committing at this time on the market. It leads both firms to the Cournot profit at time $\underline{t}$. Anticipating this result, firms forecast that they can earn more by pre-committing before this time $\underline{t}$, and so on. Finally each firm pre-commits at time $t^{*}$, the time such that the leader's profit equals firm's profit if both firms pre-committed.

8 This result is indeed more general(see [8]).

9 This loss can easily be formally computed in the linear model and it is about $10,5 \%$ of the industry profit. 


\section{$3 \quad$ Uncertainty and information spillovers}

In this section, we assume that the demand depends on a random variable. The demand is still an inverse demand function $P(Q)=\bar{a}+\eta-b$. $Q$ where $\eta$ is a random variable which follows a distribution with a zero mean and a standard deviation of $\sigma$. The market is thus uncertain. Moreover, $\eta$ may be low enough to verify $\bar{a}+\eta<c$ (i.e. the market may not be profitable). This assumption is made in order to increase the information value on demand, though the results still hold without this assumption. Firms are assumed risk neutral.

We assume that, when there is a leader on the market, there are information spillovers. For simplicity's sake the information on demand is assumed to be publicly known after the leader's production time. If there is no leader then both firms produce the Cournot quantities with $\eta$ being an unknown random variable. Thus, $\eta$ represents exactly the market information spillovers between a leader and a follower and $\sigma$ measures the importance of these information spillovers ${ }^{10}$.

These information spillovers may happen for several reasons:

First, followers are usually able to learn from past markets or leader's actions. For example, we assume that the market clears only once but markets usually clear before time 0 with a first clearing session of smaller size compared to the last one ${ }^{11}$. Typically, such a multi-period model would only be more difficult to express, whereas our simplifying assumption renders this model more simple to grasp and easier to solve ${ }^{12}$.

Second, $\bar{a}+\eta$ may represent an average revenue including, for example, an unknown production cost. But past costs are sometimes publicly observable.

Let $f(x)$ denote the probability distribution, and $F(x)$ the cumulative distribution function of the random variable $\eta$. For simplicity's sake, we use $G(x)=1-F(x)$. However, after section 3.2, in order to have a tractable model, we focus on the normal distribution thus: $f(x)=\frac{1}{\sigma \cdot \sqrt{2 \pi}} \cdot e^{-\frac{x^{2}}{2 \cdot \sigma^{2}}}$. Moreover we use the characteristic function $\chi_{x>0}=\left\{\begin{array}{l}1 \text { if } x>0 \\ 0 \text { otherwise }\end{array}\right.$.

10 We could alternatively assume that there are two random variables affecting the demand function and that only one is disclosed to the follower. In this case it is clear that the standard deviation of this random variable represents the importance of the information spillovers.

11 Note that time 0 may represent an actualization of the long-term.

12 Indeed, in a multi-period model the $\Pi_{L}^{S}$ curves on figure 3 and 4 would only shift upwards, and the analysis would not be significantly changed. 
Under these assumptions, our model can be seen as one way to extend the model of [10] in a continuous time model ${ }^{13}$.

\subsection{Subgames}

When one firm decides to lead the market, it can extract some profits from its competitor. Those profits represent the preemption value. However the follower gets the advantage of being better informed on the market conditions. Because we use an actualization rate, the follower produces as late as possible, that is at time $0^{14}$.

Proposition 2. The quantity produced by the leader $q_{L}^{*}$ is given by the following implicit function (which has a unique solution):

$$
\begin{aligned}
\int_{c+b \cdot q_{L}-\bar{a}}^{+\infty} x \cdot f(x) d x= & \left(\bar{a}-2 \cdot b \cdot q_{L}^{*}-c\right) \cdot\left(2-G\left(c+b \cdot q_{L}^{*}-\bar{a}\right)\right) \\
& -2 . c .\left(\delta^{t}-1\right)
\end{aligned}
$$

The expected leader's profit is:

$$
\Pi_{L}=b \cdot \frac{\left(q_{L}^{*}\right)^{2}}{2}\left(2-G\left(c+b \cdot q_{L}^{*}-\bar{a}\right)\right)
$$

While the expected follower's profit is:

$$
\begin{aligned}
\Pi_{F}= & \frac{\left(\bar{a}-b \cdot q_{L}^{*}-c\right)}{4 . b} \cdot\left[4\left(\bar{a}-2 b \cdot q_{L}^{*}-c \delta^{t}\right)-\left(\bar{a}-3 b \cdot q_{L}^{*}-c\right) G\left(c+b \cdot q_{L}^{*}-\bar{a}\right)\right] \\
& +\frac{1}{4 . b} \int_{x>c+b \cdot q_{L}^{*}-\bar{a}} x^{2} \cdot f(x) d x
\end{aligned}
$$

Note that the information acquired by the follower through information spillovers has a feedback effect on the leader's strategy ${ }^{15}$. Thus, the more the information spillovers, the less the preemption profits. Moreover, the information asymmetry may induce the leader to overproduce and the market may not be profitable ex post, thus the follower may not enter the market.

If both firms produce at the same time, then we have a Cournot game at time $t \geq 0$. Firms only have the partial information $\bar{a}$ on the demand function. The quantities produced in a Cournot game at time $t$ are such that $q_{i}^{C}=\frac{\bar{a}-c . \delta^{t}}{3 . b} \chi_{\bar{a}-c . \delta^{t}>0 .}$ The profits are: $\Pi^{C}(t)=\frac{\left(\bar{a}-c . \delta^{t}\right)^{2}}{9 . b} \cdot \chi_{\bar{a}-c . \delta^{t}>0}$.

13 It would be problematic to assume simultaneously a continuous time market and an instantaneous revelation of information on demand after the first entry.

14 We could alternatively assume that demand is publicly known at time 0 if there was one leader on the market.

15 See [3] for a detailed explanation of this effect. 
Profits decrease with the time of production $t$. Moreover, the characteristic function is used because when the production time is too early ( $t$ large), profits can be ex post negative. Indeed, when demand is revealed, if $\eta<c . \delta^{t}-\bar{a}$, then the overall profit of firms is negative ${ }^{16}$.

\subsection{Equilibria in pure strategies}

From now on, we assume a normal distribution. Therefore, we have four parameters in our model: $\bar{a}, b, c, \sigma$. However, this number is reduced to three by normalizing the demand function. Of course the subgame perfect equilibrium depends on these parameters. First we define two different times.

Let $t^{\prime}$ be the largest time such that $\Pi_{L}^{S}\left(t^{\prime}\right)=\Pi_{F}^{S}\left(t^{\prime}\right)>0$ and let $t^{\prime}=0$ if both profits are never equal.

Let $t^{*}$ be the largest time such that $\Pi_{L}^{S}\left(t^{*}\right)=\Pi^{C}\left(t^{*}\right)>0$ and let $t^{*}=0$ if both profits are never equal.

Indeed, in both cases, the functions only intersect once when they intersect:

It is straightforward to show that the Cournot profit is a continuous decreasing function of $t$. Moreover, in the Stackelberg case, the leader's profit is a continuous decreasing function of the production time while the follower's profit is continuous and increasing with the leader's production time. Thus, if $t$ exists such that $\Pi_{L}^{S}(t)=\Pi_{F}^{S}(t)$ then $t$ is unique.

If we start from the time $+\infty$ and if time decreases to zero, then the Cournot profit and the Stackelberg leader's one are increasing while the Stackelberg follower's profit decreases. Thus, if leading the market pays more than producing the Cournot quantities then committing later would be even better than a Cournot game at the same time. Indeed, when the pre-commitment value exceeds its cost at a time $t$, then it will exceed it also at any inferior time. Therefore, $t$ such that $\Pi_{L}^{S}(t)=\Pi^{C}(t)$ is also unique when it exists.

Hence, the leader's profit is decreasing while the follower's profit is increasing with $\sigma$ and the times $t^{\prime}$ and $t^{*}$ are decreasing with $\sigma^{17}$.

The following proposition concerns a normal distribution even though the results might hold in many other cases.

16 Note that we still assume that firms always sell their products, which means that we implicitly assume that the probability that $a+\eta<0$ is close to zero.

17 Note that the leader's profit would not depend on $\sigma$ if we were assuming that the market is always profitable whatever the quantity produced by the leader. 
Proposition 3. When $\sigma \rightarrow+\infty$, the leader never produces whatever $t$. Thus the equilibrium leads both firms to produce the Cournot quantities at time 0 .

When there are no information spillovers (that is $\sigma \approx 0$ ), each quantity produced and each profit tend to those of the previous case with no uncertainty on the demand function. Thus, by continuity, for $\sigma \approx 0$, both firms choose to produce at the same time $t^{*}$ such that $\Pi_{L}^{S}\left(t^{*}\right)=\Pi^{C}\left(t^{*}\right)$.

When uncertainty is really high, no firm wants to pre-commit on the market as none of them is willing to incur all the costs if the market appears to be not profitable. Indeed, the risks are better shared in the Cournot game at stage 0 . However, the value of information is really high in this case and the equilibrium may be suboptimal from the surplus point of view. As uncertainty decreases, a leader earns more from pre-committing, but a follower may earn even more, so that no one wants to lead the market. The situation changes when the leader can at some time earn more than in a Cournot game at time 0 .

When uncertainty is very low, the value of information is not sufficient to obtain a sequential move as an equilibrium. In this case, both firms are still trapped in a preemption race and the threat of pre-commitment forces firms to produce too soon. The next lemma and the next section illustrate the intermediate case.

Lemma 1. If $t^{\prime}$ is such that $\Pi_{L}^{S}\left(t^{\prime}\right)=\Pi_{F}^{S}\left(t^{\prime}\right)<\Pi^{C}\left(t^{\prime}\right)$, then the subgame perfect equilibrium is such that both firms produce the Cournot quantities at time $t^{*}$.

Therefore if, at time $t^{\prime}$, the Cournot outcome Pareto dominates the Stackelberg outcome, then firms wait and see until leading and committing together imply the same payoff. We have in this case a preemption race which partly dissipates the Cournot profits with no pre-commitment. Moreover, we know that this subgame perfect equilibrium is unique.

\subsection{Sequential entry}

We now characterize the cases where the equilibrium corresponds to a sequential move of firms. Figure 4 displays an example ${ }^{18}$.

We can easily infer from Fig. 4 which equilibrium will occur. Let's start for a time $+\infty$ and decrease time to zero. As time decreases until $t^{*}$, no firm wants to enter the market as it still can earn more by waiting. We have on this area a 'waiting game'. When $t^{*}>t>t^{\prime}$, then a leader on the market earns more than if both firms had produced simultaneously. However, each firm would prefer to be the follower and free ride on the information spillovers of the leader. If $t^{\prime}>t$, the leader earns more than the fol-

18 It corresponds to a case where $(\bar{a}, b, c, \sigma, \delta)=(10,1,2,3.3,1.05)$. 
lower; thus each firm wants to enter the market. In this area we have a 'preemption game'. Finally, the equilibrium is such that one firm produces at time $t^{\prime}$. It is stable as the follower has no incentive to produce at the same time (the Cournot profit being lower than the follower's one).

Figure 4. Endogenous Stackelberg case

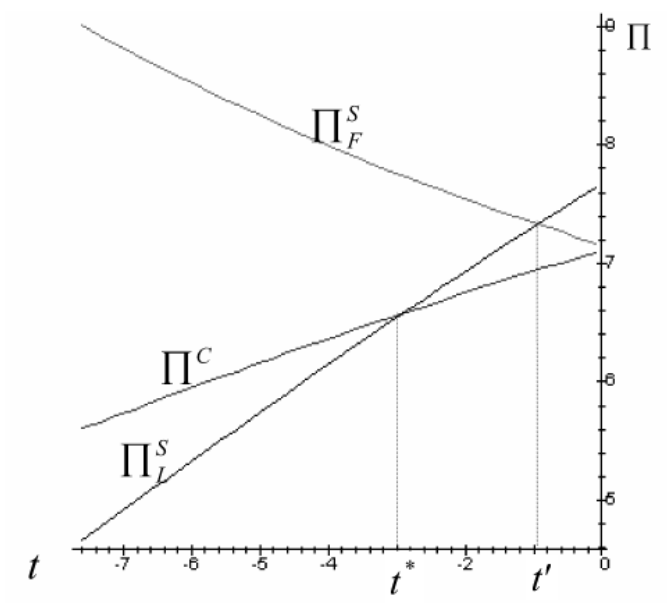

Lemma 2. If $t^{\prime}$ is such that we have $\Pi_{L}^{S}\left(t^{\prime}\right)=\Pi_{F}^{S}\left(t^{\prime}\right)>\Pi^{C}\left(t^{\prime}\right)$, then a sequential move is a subgame perfect equilibrium: the leader produces on the market at time $t^{\prime}$ and the follower produces at time 0 .

This equilibrium, as any asymmetric equilibrium in a game with symmetric players, exhibits a strong coordination problem. We have a 'rent equalization': as in [7] the leader earns the same as the follower, but the result of the leader's rent dissipation no longer holds with information spillovers. In this equilibrium, the leader preempts some of its competitor market while the follower benefits from a better information on the market conditions.

The final proposition sums up the results:

Proposition 4. If $0 \leq t^{*}<t^{\prime}$, then both firms pre-commit at time $t^{*}$ and there is a pre-emption race if $t^{*}>0$.

If $0<t^{\prime}<t^{*}$, then we have an endogenous Stackelberg where the leader pre-commits at time $t^{\prime}$ and the follower produces at time 0 while knowing the information on demand.

If $0=t^{\prime}<t^{*}$, then there is no equilibrium in pure strategies.

The relative positions between $t^{\prime}$ and $t^{*}$ depends on the parameters of the model. For example, the endogenous Stackelberg happens when the relative 
variance of the probability distribution is high enough compared to $\bar{a}$. In the next section, we analyze how the equilibrium might change with respect to the parameter $\sigma$.

\section{How information spillovers affect equilibrium}

In this model, $\sigma$ is the standard deviation of the fixed part of the demand function. As $\sigma$ increases the information gap between a leader and a follower increases, thereby $\sigma$ may be interpreted as the quantity of information spillovers between a leader and a follower. The value of $\sigma$ is decisive for the type of equilibrium in the model.

Changes in $\sigma$ affect the different profits (see the previous propositions). According to the preceding results concerning the shapes of the profits' curves, we can infer the nature of the equilibrium depending on $\sigma$.

First, as $\sigma$ increases, one can note that $q_{L}$ diminishes more than $q_{C}$ because in a Stackelberg game the follower has higher second mover advantages due to the informational aspects. Hence, the leader's profit decreases more than the Cournot profits at time $t$. This proves that $\frac{\partial t^{*}}{\partial \sigma}<0$.

Second, as $\sigma$ increases, we already proved that $\Pi_{L}^{S}$ shifts down while $\Pi_{F}^{S}$ shifts up. Therefore, this proves that $\frac{\partial t^{\prime}}{\partial \sigma}<0$.

When there are no information spillovers $(\sigma \approx 0)$, we are typically in the case of figure $3^{19}$. Hence $0<t^{*}<t^{\prime}$, firms fear pre-commitment and this leads them to produce early: there is a preemption race and firms produce the Cournot quantities at the time where leading and producing together result in the same profits. This case remains for low information spillovers.

Both $t^{*}$ and $t^{\prime}$ are decreasing with respect to $\sigma$. But, depending on the other parameters either $t^{\prime}$ decreases faster than $t^{*}$ or not. In the first case, there might exist $\sigma^{*}$ such that $0<t^{\prime}=t^{*}$ and afterwards it exists $\sigma^{\prime}$ such that $0=t^{\prime}<t^{*}$. In both cases, $t^{*}$ continues to decrease when $\sigma$ increases: there is thus a threshold $\sigma^{\prime \prime}$ such that $t^{*}=0$ and henceforth there is no incentive to lead the market.

We can easily compute the equilibria using the proposition 4 . Note that when parameters are such that $\sigma^{\prime}$ exists, we may have no equilibrium in pure strategies. Indeed, the profit functions are not upper hemi-continuous with respect to $t$ as $t$ decreases to zero and the existence results of [5] for mixed strategy equilibrium do not even apply.

19 As firms are risk neutral, if firms have the same information when producing, then firms almost act as in the model with perfect information on the expected level of demand. 
The following table ${ }^{20}$ displays how equilibrium depends on the amount of information spillovers for the most common cases:

\begin{tabular}{|l|l|l|l|l|}
\hline $\begin{array}{c}\text { Information } \\
\text { Spillovers }\end{array}$ & \multicolumn{1}{|c|}{$0 . . \sigma^{*}$} & \multicolumn{1}{|c|}{$0 . . \sigma^{*}$} & $\sigma^{\prime} . . \sigma^{\prime \prime}$ & \multicolumn{1}{c|}{$\sigma^{\prime \prime} \ldots \infty$} \\
\hline $\begin{array}{l}\text { Relative } \\
\text { position }\end{array}$ & $0<t^{*}<t^{\prime}$ & $0<t^{\prime}<t^{*}$ & $0=t^{\prime}<t^{*}$ & $0=t^{*} \leq t^{\prime}$ \\
\hline Entry profile & $\left(t^{*}, t^{*}\right)$ & $\left(t^{\prime}, 0\right) ;\left(0, t^{\prime}\right)$ & None & $(0,0)$ \\
\hline $\begin{array}{l}\text { Equilibrium in } \\
\text { pure strategies }\end{array}$ & $\begin{array}{l}\text { Preemption } \\
\text { race }\end{array}$ & $\begin{array}{l}\text { Sequential } \\
\text { entry }\end{array}$ & $\begin{array}{l}\text { No } \\
\text { equilibrium }\end{array}$ & $\begin{array}{l}\text { No } \\
\text { Precommitment }\end{array}$ \\
\hline
\end{tabular}

For example, when parameters are such that $(\bar{a}, b, c, \delta)=(10,1,2,1.05)$ then all cases happen. Therefore we may have a sequential entry as an equilibrium which increases both firms' profits for intermediate values of information spillovers.

In our model with information spillovers, we no longer have the leader's rent dissipation whenever the Stackelberg output Pareto dominates the Cournot one at the same time. Moreover, we know that the average total quantity produced in a Stackelberg game is higher than in a Cournot game. Hence, a sequential move can enhance the total surplus as well and there might be an optimal quantity of information spillovers that maximizes the total surplus. We can conclude that the existence of information spillovers can shift the equilibrium from a costly preemption race to a Pareto-superior sequential entry. Hence, even leaders sometimes end up benefiting from the existence of information spillovers ${ }^{21}$.

\section{Conclusion}

The ability to pre-commit on a market may be harmful to firms. Indeed, the threat of competitors may induce firms to produce early on emerging market while it is usually more costly. Firms face a preemption race and hence commit to market too soon. However, this pre-commitment tendency is soften when there are information spillovers between firms.

In many situations the first incumbent, by its mere choices, disclose pieces of information on the market conditions to its competitor. This creates

20 Depending on $a$ and $b$, some intervals of $\sigma$ may not exist.

21 This result states that the transparency on past moves on a market are important. While the result about the existence of an optimal quantity of information spillovers mitigates somehow this last sentence. 
value for a sequential entry which can be a subgame perfect equilibrium when these information spillovers are intermediate. When information spillovers are low, we still have a preemption race. For really high information spillovers, the information value on demand is high and a sequential entry is better for the industry surplus. However, the leader bears all the risk of the market, and thus no one wants to pre-commit. This seems to indicate that subsidies to induce early entries are likely to be efficient on highly risky markets.

The result of [7] of rent equalization still holds because, even when the equilibrium is asymmetric on the production time, the ex ante expected equilibrium payoffs are identical for both firms. In fact, the value created by the information spillovers is shared between firms via the preemption profits; this extra value also explains why the leader's rent dissipation result does not directly hold with information spillovers. Finally, all firms including the leader can benefit from the existence of information spillovers about the market conditions.

\section{A Proofs}

\section{A.1 Proof of proposition 1}

The game is a stopping game. In order to find the subgame perfect equilibrium, it suffices to study the incentives of firms on pre-committing from $t=+\infty$ to $t=0$. But first we prove three lemmas including a computation of $t^{*}$.

Lemma A Stackelberg leader is better off waiting time 0 to produce iff

$$
t>\underline{t}=\frac{1}{\ln (\delta)} \cdot \ln \left(\frac{1}{2 \cdot c} \cdot\left(a+c+\frac{2 \cdot \sqrt{2}}{3} \cdot(c-a)\right)\right)
$$

Proof.

$$
\begin{aligned}
\Pi^{C}(0) & >\Pi_{L}^{S}(t) \\
\frac{(a-c)^{2}}{9 \cdot b} & >\frac{1}{8 \cdot b} \cdot\left(a+c-2 \cdot c \cdot \delta^{t}\right)^{2} \\
8 .(a-c)^{2} & >9 \cdot\left(a+c-2 \cdot c \cdot \delta^{t}\right)^{2}
\end{aligned}
$$

As $a-c>0$ and $a+c-2 \cdot c \cdot \delta^{t}>0$, we can take the square root of this equality. It leads to:

$$
t>\frac{1}{\ln (\delta)} \cdot \ln \left(\frac{1}{2 \cdot c} \cdot\left(a+c+\frac{2 \cdot \sqrt{2}}{3} \cdot(c-a)\right)\right)
$$


Lemma When $t<\underline{t}$, a Stackelberg follower earns strictly less than a leader. Proof. This intuitive result can be formally proved:

As $\frac{(a-c)^{2}}{9 . b}<\frac{1}{8 . b} .\left(a+c-2 . c . \delta^{t}\right)^{2}$, this implies that $2 c \delta^{t}<\frac{3-2 \sqrt{2}}{3} a+\frac{3+2 \sqrt{2}}{3} c$.

Hence we can find a majoration of the follower's profit:

$$
\begin{aligned}
\Pi_{F}^{S}(t) & =\frac{1}{16 b}\left(a-3 c+2 c \delta^{t}\right)^{2} \\
& <\frac{(3-\sqrt{2})^{2}}{4} \frac{(a-c)^{2}}{9 . b} \\
& <\Pi^{C}(0)<\Pi_{L}^{S}(t)
\end{aligned}
$$

Lemma Cournot profit equal Stackelberg leader's profit for a time $t^{*}$ such that:

$$
t^{*}=\frac{1}{\ln (\delta)} \cdot \ln \left(\frac{9 \cdot c+5 \cdot a-3(a-c) \cdot \sqrt{2}}{14 \cdot c}\right)
$$

Moreover when $t<t^{*}$, the Stackelberg leader earns more than a Cournot firm (and less when $t>t^{*}$ ).

Proof. $t^{*}$ is the time such that:

$$
\begin{gathered}
\Pi^{C}(t)=\Pi_{L}^{S}(t) \\
\frac{\left(a-c \cdot \delta^{t}\right)^{2}}{9 . b}=\frac{1}{8 \cdot b} \cdot\left(a+c-2 \cdot c \cdot \delta^{t}\right)^{2} \\
\Leftrightarrow 28 \cdot c^{2} \cdot \delta^{2 t}-\left(20 \cdot a \cdot c+36 \cdot c^{2}\right) \cdot \delta^{t}+a^{2}+9 \cdot c^{2}+18 \cdot a \cdot c=0
\end{gathered}
$$

Thus $\delta^{t}$ is a root of a quadratic polynomial. Its discriminant is:

$$
\begin{aligned}
\Delta & =\left(20 \cdot a \cdot c+36 \cdot c^{2}\right)^{2}-112 \cdot c^{2} \cdot\left(a^{2}+9 \cdot c^{2}+18 \cdot a \cdot c\right) \\
& =288 \cdot(a-c)^{2} \cdot c^{2}>0
\end{aligned}
$$

There are two real positive solutions which leads to:

$$
\begin{aligned}
& t_{1}=\frac{1}{\ln (\delta)} \cdot \ln \left(\frac{9 \cdot c+5 \cdot a+3 \cdot(a-c) \cdot \sqrt{2}}{14 \cdot c}\right) \\
& t_{2}=\frac{1}{\ln (\delta)} \cdot \ln \left(\frac{9 \cdot c+5 \cdot a-3(a-c) \cdot \sqrt{2}}{14 \cdot c}\right)
\end{aligned}
$$

Let's remind that the formula defining $\Pi_{L}^{S}(t)$ is only valid for $t<\bar{t}$, where $\bar{t}$ is such that: $\bar{t}=\frac{1}{\ln (\delta)} \cdot \ln \left(\frac{a+c}{2 . c}\right)$ 
By assumption, $a>c$. Thus, we have:

$$
\begin{aligned}
(5+3 \sqrt{2}) \cdot a+(9-3 \sqrt{2}) \cdot c & =7 \cdot a+(3 \sqrt{2}-2) \cdot a+(9-3 \sqrt{2}) \cdot c \\
& >7 \cdot a+(3 \sqrt{2}-2) \cdot c+(9-3 \sqrt{2}) \cdot c \\
& >7 \cdot(a+c) \\
\frac{9 . c+5 \cdot a+3 \cdot(a-c) \cdot \sqrt{2}}{14 c} & >\frac{a+c}{2 c}
\end{aligned}
$$

Thus, as $l n$ is an increasing function, $t_{1}>\bar{t}$ and $t_{1}$ is not feasible.

$$
\begin{aligned}
(9+3 \sqrt{2}) \cdot c+(5-3 \sqrt{2}) \cdot a & =7 \cdot c+(2+3 \sqrt{2}) \cdot c+(5-3 \sqrt{2}) \cdot a \\
& <7 \cdot c+(2+3 \sqrt{2}) \cdot a+(5-3 \sqrt{2}) \cdot a \\
& <7 \cdot(a+c) \\
\frac{9 \cdot c+5 \cdot a-3 \cdot(a-c) \cdot \sqrt{2}}{14 c} & <\frac{a+c}{2 c}
\end{aligned}
$$

Thus, $t_{2}<\bar{t}$ and $t^{*}$ is uniquely defined by:

$$
t^{*}=\frac{1}{\ln (\delta)} \cdot \ln \left(\frac{9 \cdot c+5 \cdot a-3(a-c) \cdot \sqrt{2}}{14 \cdot c}\right)
$$

Moreover, if $t<t^{*}$ then $\Pi_{L}^{S}(t)>\Pi^{C}(t)$ else $\Pi_{L}^{S}(t)<\Pi^{C}(t)$

Therefore, we know that $\Pi^{C}\left(t^{*}\right)=\Pi_{L}^{S}\left(t^{*}\right)>\Pi_{F}^{S}\left(t^{*}\right)$. It is straightforward to show that the follower's profit decreases while the Cournot's profit increases when the time $t$ decreases to zero. Hence: $t^{*}>t>0 \Rightarrow \Pi^{C}(t)>\Pi_{F}^{S}(t)$. This proves that $t^{*} \geq t \geq 0 \Rightarrow \Pi_{L}^{S}(t)>\Pi^{C}(t)>\Pi_{F}^{S}(t)$. Therefore, if game starts at $t \leq t^{*}$, firms produce together at time $t$ (Cournot).

Moreover, $\underline{t}>t>t^{*} \Rightarrow 0<\Pi_{L}^{S}(t)<\Pi^{C}(t)<\Pi^{C}\left(t^{*}\right)$. Therefore, firms have no incentive to produce before $t^{*}$.

Eventually, the only subgame perfect equilibrium is when both firms produce at time $t^{*}$, such that $\Pi_{L}^{S}\left(t^{*}\right)=\Pi^{C}\left(t^{*}\right)$.

\section{A.2 Proof of proposition 2}

We compute the subgame perfect equilibrium by backward induction. The Stackelberg follower produces whenever it is profitable. He has a monopoly on the residual demand, therefore:

$$
q_{F}(\eta)=\frac{\bar{a}+\eta-b \cdot q_{L}-c}{2 . b} \cdot \chi_{\bar{a}+\eta-b . q_{L}-c>0}
$$


Anticipating this result, the leader maximizes its profit:

$$
\Pi_{L}(\eta)=\left(\bar{a}+\eta-b \cdot q_{L}-c \cdot \delta^{t}-\frac{\bar{a}+\eta-b \cdot q_{L}-c}{2} \cdot \chi_{\bar{a}+\eta-b . q_{L}-c>0}\right) \cdot q_{L}
$$

The leader's expected profit is:

$$
\begin{aligned}
\Pi_{L}= & \int_{x} \Pi_{L}(x) \cdot f(x) d x \\
\Pi_{L}= & \left(\bar{a}-b \cdot q_{L}-c \cdot \delta^{t}\right) \cdot q_{L}-\frac{\bar{a}-b \cdot q_{L}-c}{2} \cdot q_{L} \cdot G\left(c+b \cdot q_{L}-\bar{a}\right) \\
& -\frac{q_{L}}{2} \int_{x>c+b \cdot q_{L}-\bar{a}} x \cdot f(x) d x
\end{aligned}
$$

We look for the solutions of the first order condition:

$$
\begin{aligned}
\frac{\partial \Pi_{L}}{\partial q_{L}}= & \bar{a}-2 \cdot b \cdot q_{L}-c \cdot \delta^{t}-\frac{\bar{a}-2 \cdot b \cdot q_{L}-c}{2} \cdot G\left(c+b \cdot q_{L}-\bar{a}\right) \\
& -\frac{\bar{a}-b \cdot q_{L}-c}{2} \cdot q_{L} \cdot \frac{\partial}{\partial q_{L}}\left[G\left(c+b \cdot q_{L}-\bar{a}\right)\right] \\
& -\frac{\partial}{\partial q_{L}}\left[\frac{q_{L}}{2} \cdot \int_{x>c+b \cdot q_{L}-\bar{a}} x \cdot f(x) d x\right] \\
= & \frac{1}{2}\left(\bar{a}-2 \cdot b \cdot q_{L}\right) \cdot\left(2-G\left(c+b \cdot q_{L}-\bar{a}\right)\right) \\
& -c \cdot\left(\delta^{t}-\frac{1}{2} G\left(c+b \cdot q_{L}-\bar{a}\right)\right) \\
& +b \frac{\bar{a}-b \cdot q_{L}-c}{2} \cdot q_{L} \cdot f\left(c+b \cdot q_{L}-\bar{a}\right) \\
& -\frac{1}{2} \int_{x>c+b \cdot q_{L}-\bar{a}} x \cdot f(x) d x+b \frac{q_{L}}{2}\left(c+b \cdot q_{L}-\bar{a}\right) \cdot f\left(c+b \cdot q_{L}-\bar{a}\right) \\
& \frac{1}{2}\left(\bar{a}-2 \cdot b \cdot q_{L}\right) \cdot\left(2-G\left(c+b \cdot q_{L}-\bar{a}\right)\right) \\
& -\frac{1}{2} \int_{x>c+b \cdot q_{L}-\bar{a}} x \cdot f(x) d x \\
& -c \cdot\left(\delta^{t}-\frac{1}{2} G\left(c+b \cdot q_{L}-\bar{a}\right)\right)
\end{aligned}
$$

As this derivative must equal zero, we obtain $q_{L}^{*}$ as an implicit function:

$$
\begin{aligned}
\int_{c+b \cdot q_{L}-\bar{a}}^{+\infty} x \cdot f(x) d x= & \left(\bar{a}-2 \cdot b \cdot q_{L}-c\right) \cdot\left(2-G\left(c+b \cdot q_{L}-\bar{a}\right)\right) \\
& -2 \cdot c \cdot\left(\delta^{t}-1\right)
\end{aligned}
$$


When it exists, this solution is unique as the leader's profit is clearly strictly concave in $q_{L}$.

Moreover, by injecting this implicit formula in the definition of the leader's profit, one obtains a simpler formula:

$$
\Pi_{L}=b \cdot \frac{\left(q_{L}^{*}\right)^{2}}{2}\left(2-G\left(c+b \cdot q_{L}^{*}-\bar{a}\right)\right)
$$

The follower's profit is:

$$
\begin{aligned}
\Pi_{F}(\eta) & =\left(\bar{a}+\eta-b \cdot q_{L}-b \cdot \frac{\bar{a}+\eta-b \cdot q_{L}-c}{2 . b} \cdot \chi_{\bar{a}+\eta-b . q_{L}-c>0}-c\right) \cdot q_{F} \\
& =\frac{1}{2}\left(\bar{a}+\eta-b \cdot q_{L}-c\right) \cdot\left(1+\chi_{\bar{a}+\eta-b . q_{L}-c<0}\right) \cdot \frac{\bar{a}+\eta-b \cdot q_{L}-c}{2 . b} \cdot \chi_{\bar{a}+\eta-b . q_{L}-c>0} \\
& =\frac{\left(\bar{a}+\eta-b \cdot q_{L}-c\right)^{2}}{4 . b} \cdot \chi_{\eta>c+b \cdot q_{L}-\bar{a}}
\end{aligned}
$$

The ex ante follower's expected profit is therefore:

$$
\begin{aligned}
\Pi_{F}= & \int \Pi_{F}(x) \cdot f(x) d x \\
= & \frac{\left(\bar{a}-b \cdot q_{L}-c\right)^{2}}{4 \cdot b} \cdot G\left(c+b \cdot q_{L}-\bar{a}\right) \\
& +\frac{1}{2 \cdot b}\left(\bar{a}-b \cdot q_{L}-c\right) \int_{x>c+b \cdot q_{L}-\bar{a}} x \cdot f(x) d x \\
& +\frac{1}{4 \cdot b} \int_{x>c+b \cdot q_{L}-\bar{a}} x^{2} \cdot f(x) d x \\
= & \frac{\left(\bar{a}-b \cdot q_{L}-c\right)}{4 \cdot b} \cdot\left[\left(\bar{a}-b \cdot q_{L}-c\right) G\left(c+b \cdot q_{L}-\bar{a}\right)+2 \int_{x>c+b \cdot q_{L}-\bar{a}} x \cdot f(x) d x\right] \\
& +\frac{1}{4 \cdot b} \int_{x>c+b \cdot q_{L}-\bar{a}} x^{2} \cdot f(x) d x
\end{aligned}
$$

By injecting the formula defining $q_{L}^{*}$, one obtains:

$$
\begin{aligned}
\Pi_{F}= & \frac{\left(\bar{a}-b \cdot q_{L}^{*}-c\right)}{4 . b} \cdot\left[4\left(\bar{a}-2 b \cdot q_{L}^{*}-c \delta^{t}\right)-\left(\bar{a}-3 b \cdot q_{L}^{*}-c\right) G\left(c+b \cdot q_{L}^{*}-\bar{a}\right)\right] \\
& +\frac{1}{4 . b} \int_{x>c+b \cdot q_{L}^{*}-\bar{a}} x^{2} \cdot f(x) d x
\end{aligned}
$$

\section{A.2.1 Normal distribution}

Moreover concerning the normal distribution we have the following equation: $\int_{y}^{+\infty} x \cdot f(x) d x=\sigma^{2} \cdot f(y)$. Hence, the implicit function becomes: 


$$
\begin{aligned}
\sigma^{2} \cdot f\left(c+b \cdot q_{L}^{*}-\bar{a}\right)= & \left(\bar{a}-2 \cdot b \cdot q_{L}-c\right) \cdot\left(2-G\left(c+b \cdot q_{L}-\bar{a}\right)\right) \\
& -2 . c \cdot\left(\delta^{t}-1\right)
\end{aligned}
$$

The leader's profit remains:

$$
\Pi_{L}=b \cdot \frac{\left(q_{L}^{*}\right)^{2}}{2}\left(2-G\left(c+b \cdot q_{L}^{*}-\bar{a}\right)\right)
$$

The computation of the follower's expected profit uses only a simple integration by part:

$$
\begin{aligned}
\Pi_{F}= & \frac{\left(\bar{a}-b \cdot q_{L}-c\right)^{2}}{4 \cdot b} \cdot G\left(c+b \cdot q_{L}-\bar{a}\right) \\
& +\frac{1}{\sigma \cdot \sqrt{2 \pi}} \cdot \frac{\sigma^{2}}{2 \cdot b}\left(\bar{a}-b \cdot q_{L}-c\right) \cdot e^{-\frac{\left(c+b \cdot q_{L}-\bar{a}\right)^{2}}{2 \cdot \sigma^{2}}} \\
& +\frac{1}{\sigma \cdot \sqrt{2 \pi}} \cdot \frac{\sigma^{2}}{4 \cdot b} \cdot\left(\left(c+b \cdot q_{L}-\bar{a}\right) \cdot e^{-\frac{\left(c+b \cdot q_{L}-\bar{a}\right)^{2}}{2 \cdot \sigma^{2}}}+\int_{c+b \cdot q_{L}-\bar{a}}^{+\infty} e^{-\frac{x^{2}}{2 \cdot \sigma^{2}}} d x\right) \\
= & \frac{\left(\bar{a}-b \cdot q_{L}-c\right)^{2}}{4 \cdot b} \cdot G\left(c+b \cdot q_{L}-\bar{a}\right) \\
& +\frac{\sigma}{2 \cdot \sqrt{2 \pi} b}\left(\bar{a}-b \cdot q_{L}-c\right) \cdot e^{-\frac{\left(c+b \cdot q_{L}-\bar{a}\right)^{2}}{2 \cdot \sigma^{2}}} \\
& +\frac{\sigma}{4 \cdot \sqrt{2 \pi} b} \cdot\left(c+b \cdot q_{L}-\bar{a}\right) \cdot e^{-\frac{\left(c+b \cdot q_{L}-\bar{a}\right)^{2}}{2 \cdot \sigma^{2}}}+\frac{\sigma^{2}}{4 \cdot b} G\left(c+b \cdot q_{L}-\bar{a}\right)
\end{aligned}
$$

which concludes:

$$
\begin{aligned}
\Pi_{F}= & \frac{\left(\bar{a}-b \cdot q_{L}-c\right)^{2}+\sigma^{2}}{4 \cdot b} \cdot G\left(c+b \cdot q_{L}-\bar{a}\right) \\
& +\frac{\sigma^{2}}{4 b}\left(\bar{a}-b \cdot q_{L}-c\right) \cdot f\left(c+b \cdot q_{L}-\bar{a}\right)
\end{aligned}
$$

\section{A.3 Proof of proposition 3}

With the normal distribution we have the following implicit function for $q_{L}^{*}$ :

$$
\begin{aligned}
\sigma^{2} \cdot f\left(c+b \cdot q_{L}^{*}-\bar{a}\right)= & \left(\bar{a}-2 \cdot b \cdot q_{L}-c\right) \cdot\left(2-G\left(c+b \cdot q_{L}-\bar{a}\right)\right) \\
& -2 . c \cdot\left(\delta^{t}-1\right)
\end{aligned}
$$

We first notice that the left term of the implicit function defining $q_{L}^{*}$ tends to infinity when $\sigma \rightarrow+\infty$ and the right term is bounded above $\left(q_{L}\right.$ is positive). Thus, there is no solution for the first order condition. More- 
over $\frac{\partial}{\partial q_{L}} \Pi_{L}\left(q_{L}\right)$ is clearly negative for $q_{L}$ large enough, so that we can finally conclude that the constraint on the maximization problem is binding i.e. $\lim _{\sigma \rightarrow+\infty} q_{L^{*}}=0$.

When $\sigma \approx 0$, the implicit function becomes:

$$
\begin{aligned}
0 & =\left(\bar{a}-2 \cdot b \cdot q_{L}^{*}-c\right)-2 \cdot c \cdot\left(\delta^{t}-1\right) \\
q_{L}^{*} & =\frac{1}{2 . b} \cdot\left[\bar{a}+c-2 . c \cdot \delta^{t}\right]
\end{aligned}
$$

\section{A.4 Proof of lemma 1}

First we remark that, in this case, $t^{\prime}>t^{*} \geq 0 . \Pi_{F}^{S}$ decreases while $\Pi^{C}$ increases when the time, $t$, decreases to zero. Therefore: $t^{\prime}>t \geq 0 \Rightarrow \Pi_{F}^{S}(t)<\Pi^{C}(t)$. This means that, for any time smaller than $t^{\prime}$, firms prefers to produce simultaneously rather than being a follower. Moreover, $t^{*}>t \geq 0 \Rightarrow$ $\Pi_{L}^{S}(t)>\Pi^{C}(t)$. Hence for any smaller time than $t^{*}$, firms are prompted to produce and there is a preemption race for $t<t^{*}$. Finally, the equilibrium is such that both firms produce simultaneously on the market at time $t^{*}$.

\section{A.5 Proof of lemma 2}

First we remark that, in this case, $t^{*}>t^{\prime} \geq 0$. We know that $\Pi_{F}^{S}$ decreases while $\Pi_{L}^{S}$ increases when the time decreases to zero. Thus: $t>t^{\prime} \Rightarrow \Pi_{F}^{S}(t)>$ $\Pi_{L}^{S}(t)$. We have a Waiting game: each firm would like the other to lead the market. On the contrary: $t^{\prime}>t \geq 0 \Rightarrow \Pi_{L}^{S}(t)>\Pi_{F}^{S}(t)$ and each firm prefers to preempt the market than being preempted. The follower earns more by waiting than by producing together with the leader (i.e. $\left.\Pi_{F}^{S}\left(t^{\prime}\right)>\Pi^{C}\left(t^{\prime}\right)\right)$. Hence, the subgame perfect equilibrium is such that one firm produces at time $t^{\prime}$ and the other waits time 0 to produce.

\section{A.6 Proof of proposition 4}

If $t^{*}=0$ then, at any time $t>0$, the leader's profit is always lower than the Cournot profit at time $t$. Firms have no incentive to lead the market and the subgame perfect equilibrium is such that both firms end up producing the Cournot quantity at time 0 .

If $0 \leq t^{*}<t^{\prime}$, we know that: $t>t^{*} \Rightarrow \Pi_{L}^{S}(t)<\Pi^{C}(t)$. Thus $\Pi_{F}^{S}\left(t^{\prime}\right)=$ $\Pi_{L}^{S}\left(t^{\prime}\right)<\Pi^{C}\left(t^{\prime}\right)$ and we can apply lemma 1 which concludes. 
If $0<t^{\prime}<t^{*}$, we know that: $t<t^{*} \Rightarrow \Pi_{L}^{S}(t)>\Pi^{C}(t)$. Thus $\Pi_{F}^{S}\left(t^{\prime}\right)=$ $\Pi_{L}^{S}\left(t^{\prime}\right)>\Pi^{C}\left(t^{\prime}\right)$ and we can apply lemma 2 which concludes.

If $0=t^{\prime}<t^{*}$, we initially notice that, for $t>T$ sufficiently large, $\Pi_{F}^{S}(t)>\Pi^{C}(t)$. Indeed, when $t$ is too large, the leader does not produce. Hence the follower earns more than a Cournot player as it is monopolizing the market. As $t^{\prime}=0$ and $\Pi_{F}^{S}(T)>\Pi_{L}^{S}(T)$, we conclude by continuity that $\forall t>0: \Pi_{F}^{S}(t)>\Pi_{L}^{S}(t)$. Thus: $t^{*}>t \geq 0 \Rightarrow \Pi_{F}^{S}(t)>\Pi_{L}^{S}(t)>\Pi^{C}(t)$. First, leading the market at a time $t<t^{*}$ is a dominated strategy, as leading the market at time $\frac{t}{2}$ leads to a higher profit while the follower does not move to this deviation. Second, if firms produce together at time $t$, then it is optimal to lead the market at time $t+\varepsilon$. Thus, a Cournot output at time $t$ cannot be an equilibrium. Hence we conclude that there is no equilibrium in pure strategy in this case.

\section{References}

[1] Boyer, M. and M. Moreaux (1987), "Being a Leader or a Follower: Reflections on the Distribution of Roles in Duopoly", International Journal of Industrial Organization, vol.5, pp. 175-192.

[2] Boyer, M. and M. Moreaux (1989), "Uncertainty, Capacity and Flexibility: the Monopoly case", Annales d'Economie et de Statistiques vol.15/16, pp. 291-313.

[3] Caron, C. and T. Lafay (2008), "How Risk Disciplines Pre-commitment", Theory and Decision, vol.65(3), pp. 205-226.

[4] Cournot, A.A. (1838), Recherches sur les principes mathématiques de la théorie des richesses, Paris, Hachette.

[5] Dasgupta, P. and E. Maskin, (1986), "The existence of equilibrium in discontinuous economic games I: theory; II: applications", Review of Economic Studies, vol.53, pp. 1-41.

[6] D'Aspremont, C., Gabszewicz, J., Thisse, J.F., (1979), "On Hotelling's 'Stability in Competition"', Econometrica, vol.47, pp. 1145-1150.

[7] Fudenberg, D. and J. Tirole, (1985), "Preemption and Rent Equalization in the Adoption of New Technology", Review of Economic Studies, vol.52, pp. 383-401.

[8] Fudenberg, D. and J. Tirole, (1987), "Understanding Rent Dissipation: On the Use of Game Theory in Industrial Organization", The American Economic Review, vol.172, pp. 176-183.

[9] Hamilton, J. and S. Slutsky, (1990), "Endogenous timing in duopoly games : Stackeleberg or Cournot Equilibria", Games and Economic Behavior, vol.2, pp. 29-46.

[10] Hirokawa, M. and D. Sasaki, (2001), "Endogenously Asynchronous Entries into an Uncertain Industry", Journal of Economics and Management Strategy, vol. 10(3), pp. $435-461$. 
[11] Maggi, G., (1996), "Endogenous Leadership in a New Market", RAND Journal of Economics, vol.27, pp. 641-659.

[12] Pacheco-de-Almeida, G. and P. Zemsky, (2003), "The Effect of Time-to-Build on Strategic Investment Under Uncertainty", RAND Journal of Economics, vol.34, pp. 166-182.

[13] Porter, M. E., (1998), "Clusters and the new economics of competition", Harvard Business Review, vol.76(6), pp. 77-90.

[14] Spencer, B. and J. Brander, (1992), "Pre-commitment and flexibility : applications to oligopoly theory", European Economic Review, vol.36, pp. 1601-1626.

[15] von Stackelberg, H., (1934), Marktform und Gleichgwicht, Vienna and Berlin, Springer Verlag.

[16] van Damme, E. and S. Hurken, (1999), "Endogenous Stackelberg Leadership", Games and Economic Behavior, vol.28, pp. 105-129. 